\title{
Composite Criticality in Machinery Fleet Management of Construction Projects
}

\author{
John-Paris Pantouvakis ${ }^{1 *}$, and Alexander Maravas ${ }^{1}$ \\ ${ }^{1}$ Centre for Construction Innovation, Department of Construction Engineering and $\mathrm{M}$ anagement, \\ Faculty of Civil Engineering, National Technical University of A thens, Greece
}

\begin{abstract}
During construction operations, fleet management aims at maximizing the uptime and efficiency of construction machinery while also minimizing the cost of ownership through lifecycle planning and management. In the deterministic approach, the theory suggests that one type of machinery is considered to be critical. However, taking into account the real circumstances under which projects are performed with issues such as machine reliability, worker performance, and/or errors in estimating the scope of work, it is evident that there are significant limitations to the existing approach. Hence, to address this issue, uncertainty in fleet productivity is modelled with fuzzy set theory. In this context, the notion of composite criticality under which the productivity of a fleet depends on more than one type of machinery because of the fluctuations of the individual productivities is introduced. To this purpose, a simple case study is presented to illustrate this concept. It is concluded that this approach leads to a better understanding of the estimation of activity duration and cost estimation which in turn means better project scheduling and financial planning.
\end{abstract}

Keywords: construction management, fleet management, fuzzy systems, financial planning, uncertainty management.

\section{Introduction}

In construction operations project managers are faced with the task of completing activities under multiple constraints while ensuring the optimal use of available machinery. In this context, construction equipment fleet management involves a variety of functions including machine maintenance, safety audits, GPS tracking, optimal fleet composition, cost tracking, fuel consumption monitoring and operator training. In ensuring efficiency, a primary goal is to maximize machinery uptime and reduce idle times by ensuring continuous flow and usage of machinery in all project activities. Similarly, managers seek to minimize rental or ownership costs by mobilizing the optimal number of necessary machinery.

* Corresponding author: jpp@ cental.ntua.gr 
Productivity estimation remains a difficult task to be performed at the pre-construction stage [1]. For many years the deterministic approach has dominated the methodological approach to the estimations of machine productivity and consequently fleet composition. In construction operations, productivity theory and performance handbooks provide an excellent base for estimating the productivity of construction equipment. Thereafter, in determining fleet composition, the primary assumption is that in a group of machines the productivity of the machinery with the lowest productivity will determine the total production capability. However, productivity rates differ between projects because of the varying environments, characteristics, and level of management implementation [2]. Also, taking into account issues such as productivity, machine reliability, worker performance, and/or errors in estimating the scope of work, it is evident that there are significant limitations to the existing approach. Finally, the study of a fleet of machinery in which there is uncertainty in the productivity of its individual components poses a significant challenge.

The purpose of this paper is to apply a fuzzy set theory to examine issues of uncertainty and imprecision in machinery fleet composition. Initially, uncertainty is examined on the productivity of individual machines. Thereafter, the notion of composite criticality is introduced, under which the productivity of a fleet of machines depends on more than one type of machinery because of the fluctuations of the individual productivities. The concept is exemplified in an earthworks project. Finally, the conclusions and possibilities for future research are presented.

\section{Literature Review}

\subsection{Performance Handbooks}

Performance handbooks, provided by the manufacturers of construction machinery have assisted project managers in estimating productivity and optimizing fleet composition. The Caterpillar Performance Handbook [3] includes productivity estimations and guidelines for a variety of equipment such as tractors, graders, loaders, excavators, backhoe loaders, forest machines, pipelayers, scrapers, trucks, landfill compactors, pavers and asphalt pavers. The Performance Handbook serves as an aid which, when coupled with experience can assist in estimating true machine performance and optimizing fleet composition. Similarly, the Specifications and Applications Handbook [4] has extensive guidelines on productivity estimation. Overall, performance handbooks combined with productivity theory as in Peurifoy [5] and Nunally [6] provide an excellent basis for productivity analysis.

\subsection{Match Factor}

In earthworks activities, Douglas [7] published a formula based on the ratio of loader productivity to truck productivity that can be used to determine the optimal number of trucks to balance shovel output. Morgan and Peterson [8] introduced the concept of the match factor which is very useful in providing a measure of the productivity of a fleet and quite similar to the Douglas ratio. A match factor of 1 represents a balance point where trucks are arriving at the loader at the same time they are being served. If the ratio exceeds 1 , the trucks are arriving faster than they are being served and a queue is formed. If the ratio is below 1 , the loaders are serving faster than the trucks arriving [9]. In theory, a match factor of 1 is an indication of a maximized productivity level. 


\subsection{Fuzzy Set Theory}

Fuzzy Set Theory is used to describe and quantify uncertainty and imprecision in data and functional relationships. A fuzzy subset $\mathrm{A}$ of a universe of discourse $\mathrm{U}$ is characterized by a membership function $\mu_{A}: U \rightarrow[0,1]$ which associates with each element $x$ of $U$ a number $\mu_{\mathrm{A}}(\mathrm{x})$ in the interval $[0,1]$ which represents the grade of membership of $\mathrm{x}$ in A. In fuzzy set theory, the triangular membership function which is defined by three numbers $a, b, c$ is frequently utilized. As such, a triangular fuzzy number $\tilde{x}=\langle a, b, c\rangle$ has the following membership function:

$$
\mu_{A}(x)=\left\{\begin{array}{l}
0, \quad x<a \\
(x-a) /(b-a), \quad a \leq x \leq b \\
(c-x) /(c-b), b \leq x \leq c \\
0, \quad x>c
\end{array}\right.
$$

The rules that determine the operations of addition, subtraction, multiplication and division of fuzzy numbers are called fuzzy arithmetic [10].

The $\min (\wedge)$ and $\max (\vee)$ operators are useful in finding the maximum or minimum of two membership functions [10].

$$
\begin{aligned}
& \mu_{A(\wedge) B}(z)=\bigvee_{z=x \wedge y}\left(\mu_{A}(x) \wedge \mu_{B}(y)\right) \\
& \mu_{A(\vee) B}(z)=\bigvee_{z=x \vee y}\left(\mu_{A}(x) \vee \mu_{B}(y)\right)
\end{aligned}
$$

As such the union and the intersection of two fuzzy sets are determined as:

$$
\begin{aligned}
& \mu_{\mathrm{A} \cup \mathrm{B}}(x)=\mu_{\mathrm{A}}(x) \vee \mu_{\mathrm{B}}(x)=\max \left\{\mu_{\mathrm{A}}(x), \mu_{\mathrm{B}}(x)\right\} \\
& \mu_{\mathrm{A} \cap \mathrm{B}}(x)=\mu_{A}(x) \wedge \mu_{B}(x)=\min \left\{\mu_{\mathrm{A}}(x), \mu_{\mathrm{B}}(x)\right\}
\end{aligned}
$$

In describing linguistic variables defined by fuzzy sets, Zadeh [11] proposed that they can be characterized either by atomic terms (words which always occur together) or composite terms that contain one or more atomic terms. Furthermore, he defined formula for calculating composite truth variables based on the min operator. As such, the interpretation of a composite of two fuzzy sets (such as $\mu_{\mathrm{A}}$ and $\mu_{\mathrm{B}}$ ) is based on the fuzzy min operator [12]. In applying fuzzy set theory in project scheduling, McCahon and Lee [13] explained the difference between the comparison method and the composite method in studying two fuzzy variables. The former compares fuzzy numbers based on metrics and considers one as being larger than the other, while the latter forms a new fuzzy number from pieces of the component fuzzy numbers. In this paper, the composite of two fuzzy sets refers to creating a new fuzzy set from parts of the component fuzzy sets.

\section{Productivity Analysis Model}

The purpose of the model is to utilize fuzzy set theory to represent uncertainty in the estimation of machinery productivity and the process of fleet composition based on Performance Handbooks and traditional productivity theory. Additionally, a fuzzy version of the well-established match factor is presented. 


\subsection{Machine Productivity}

In the case of a construction project that involves earthworks, the fuzzy productivity of a single excavator can be calculated with the formula [14]:

$$
\tilde{Q}_{e x}=\frac{60 \cdot V_{e} \cdot \tilde{f}}{\tilde{t}_{s}} \cdot \tilde{\eta}_{e x}
$$

where $\tilde{Q}_{e x}$ : the fuzzy production rate of an excavator in $\mathrm{m}^{3} / \mathrm{h}, 60$ : a conversion factor, $V_{e}$ : the crisp bucket capacity in $\mathrm{m}^{3}, \tilde{f}$ : the fuzzy bucket fill factor, $\tilde{t}_{s}$ : the excavator cycle time in the min and $\tilde{\eta}_{e x}$ : the operational efficiency.

Similarly, the fuzzy productivity of a truck can be calculated by the formula [15]:

$$
\tilde{Q}_{t r}=\frac{60 \cdot \tilde{V}_{t r a n s}}{\widetilde{t}_{t r}} \cdot \tilde{\eta}_{t r}
$$

where $\tilde{Q}_{t r}$ : the fuzzy production rate of a truck in $\mathrm{m}^{3} / \mathrm{h}, \tilde{V}_{\text {trans }}$ : the fuzzy volume of the material to be transferred in $\mathrm{m}^{3}, \tilde{t}_{t r}$ : the fuzzy cycle time of the truck in the min and $\tilde{\eta}_{t r}:$ the operational efficiency.

Additionally, the fuzzy cycle time of the truck is given by the formula:

$$
\tilde{t}_{\text {tr }}=\tilde{t}_{\text {mov }}+\widetilde{t}_{d u}+\widetilde{t}_{r e}+\widetilde{t}_{\text {man }}+\widetilde{t}_{\text {load }}
$$

where $\tilde{t}_{m o v}$ : the fuzzy time for moving to the dump area in min, $\tilde{t}_{d u}$ : the fuzzy time for dumping in min, $\tilde{t}_{r e}$ : the fuzzy time for returning to the load area in min, $\tilde{t}_{\text {man }}$ : the fuzzy time for maneuvering in the min and $\tilde{t}_{\text {load }}$ : the fuzzy time for loading in min.

Finally, the total required fuzzy production rate of a worksite can be calculated as:

$$
\tilde{Q}_{w s}=\frac{\tilde{V} \cdot \tilde{\varepsilon}}{t \cdot \tilde{\eta}}
$$

where $\tilde{Q}_{w s}$ : the fuzzy productivity of the worksite in $\mathrm{m}^{3} / \mathrm{h}, \tilde{V}$ : the volume of earthworks in $\mathrm{m}^{3}, \widetilde{\varepsilon}:$ the fuzzy swell factor, $t$ : the contractual completion time in hrs and $\tilde{\eta}:$ the operational efficiency.

\subsection{Fleet Composition and Productivity Analysis}

After estimating the uncertainty in trucks and excavators, fleet composition requires to find the optimal combination of these two types of construction equipment in order to meet project constraints. A deterministic analysis would state that in the case that the excavators are critical it be acceptable for the trucks to form queues. Similarly, if the trucks are critical, then the excavators will have periods that they are not able to load the trucks. If we apply fuzzy set theory to traditional fleet composition we have the following equations [15]: 
Excavators are Critical

$$
\begin{gathered}
n_{e x}=\operatorname{int}\left(\left(\tilde{Q}_{w s} / \widetilde{Q}_{e x}+1\right)\right) \\
\tilde{Q}_{\text {fleet }}=\widetilde{Q}_{E X}=n_{e x} \cdot \widetilde{Q}_{e x} \\
n_{t r}=\operatorname{int}\left(\left(\tilde{Q}_{\text {fleet }} / \widetilde{Q}_{t r}+1\right)\right) \\
\tilde{Q}_{T R}=n_{t r} \cdot \tilde{Q}_{t r}
\end{gathered}
$$

Trucks are critical

$$
\begin{gathered}
n_{t r}=\operatorname{int}\left(\left(\widetilde{Q}_{w s} / \widetilde{Q}_{t r}+1\right)\right) \\
\tilde{Q}_{\text {fleet }}=\widetilde{Q}_{T R}=n_{t r} \cdot \widetilde{Q}_{t r} \\
n_{\text {ex }}=\operatorname{int}\left(\left(\tilde{Q}_{\text {fleet }} / \tilde{Q}_{e x}+1\right)\right) \\
\tilde{Q}_{E X}=n_{e x} \cdot \widetilde{Q}_{e x}
\end{gathered}
$$

where $\widetilde{Q}_{E X}$ : the fuzzy productivity of all excavators in the fleet, $\tilde{Q}_{e x}$ : the fuzzy productivity of a single excavator, $\widetilde{Q}_{T R}$ : the fuzzy productivity of all trucks in the fleet, $\widetilde{Q}_{t r}$ : the fuzzy productivity of a single truck, $\tilde{Q}_{w S}$ : the required productivity of the worksite, $\tilde{Q}_{\text {fleet }}:$ the fuzzy productivity of the fleet of excavators and trucks, $n_{t r}:$ the number of trucks, $n_{e x}$ : the number of excavators, int: the integers in a fuzzy set.

Applying equations 10-17 along with the underlying assumption that one type of machines is always critical yields values for $n_{e x}, n_{t r}, \widetilde{Q}_{E X}$ and $\tilde{Q}_{T R}$. Similarly, $\widetilde{Q}_{\text {fleet }}$ should be the minimum of the membership functions $\mu_{E X}(x)$ of the excavator productivity ( $\left.\tilde{Q}_{E X}\right)$ and $\mu_{T R}(y)$ of the truck productivity $\left(\widetilde{Q}_{T R}\right)$. Thus, applying the minimum operator [15]:

$$
\widetilde{Q}_{\text {fleet }}=\mu_{E X}(\wedge)_{T R}(z)=\underset{z=x \wedge y}{\vee}\left(\mu_{Q_{E X}}(x) \wedge \mu_{Q_{T R}}(y)\right)
$$

In applying this formula, the membership function of the fleet productivity is not necessarily a triangular fuzzy number but in some cases a composite function that is derived from the membership function of the productivities of two or more type of machinery. In this context, the notion of composite criticality is defined as the situation in which the productivity of a fleet depends on more than one types of machinery because of the fluctuations and uncertainties of the individual productivities. Hence, the composite of two fuzzy productivity sets, form a new fuzzy set (from parts of the component fuzzy sets) that defines the criticality of the fleet.

In direct accordance with the deterministic analysis, the fuzzy match factor $(M \widetilde{F})$ is a measure for comparing the productivity of two types of machines. In the case of an earthworks project involving excavators and trucks it can be calculated as:

$$
M \tilde{F}=\widetilde{Q}_{E X} / \widetilde{Q}_{T R}
$$


Furthermore, the analysis of the fleet productivity $\tilde{Q}_{\text {fleet }}$ is much more intuitive with the fuzzy agreement index. Hence, the Agreement Index of the fleet $\left(\mathrm{AI}_{\text {fleet }}\right)$ is $\operatorname{AI}\left(\widetilde{Q}_{E X}\right.$, $\widetilde{Q}_{T R}$ ) of $\widetilde{Q}_{E X}$ in relation to $\widetilde{Q}_{T R}$ and is calculated as follows:

$$
A I_{\text {fleet }}=A I\left(\tilde{Q}_{E X}, \tilde{Q}_{T R}\right)=\left(\operatorname{area} \tilde{Q}_{E X} \cap \tilde{Q}_{T R}\right) /\left(\text { area } \tilde{Q}_{T R}\right)
$$

\section{Example}

\subsection{Excavator and Truck Productivity}

Consider an excavator manufactured by Caterpillar with a bucket capacity of $3 \mathrm{~m}^{3}$, a bucket fill factor of $\langle 0.81,0.83,0.84\rangle$, a cycle time of $\langle 0.51,0.52,0.54\rangle \mathrm{min}$ and operational efficiency of $\langle 0.78,0.80,0.81\rangle$. As shown in Table 1 , the direct application of eq. 6 yields a fuzzy productivity estimate of $\tilde{Q}_{e x}=\langle 210,230,240\rangle \mathrm{m}^{3} / \mathrm{h}$. Similarly, for a Caterpillar truck, using the fuzzified parameters of productivity from the Performance Handbook, where the volume of transferred material is $\langle 17.8,18.0,18.2\rangle \mathrm{m}^{3}$, the cycle time is $\langle 17.5,18,18.5\rangle$ min and the operational efficiency is $\langle 0.69,0.75,0.81\rangle$ then the fuzzy truck production rate will be $\langle 40,45,50\rangle \mathrm{m}^{3} / \mathrm{h}$ (Table 2 ). The cycle time estimate is calculated by applying a $3 \%$ uncertainty to the respective crisp times i.e.

$$
\begin{gathered}
t_{t r}=t_{\text {mov }}+t_{d u}+t_{r e}+t_{\text {man }}+t_{\text {load }}=8+1+4+1+4=18 \mathrm{~min} \\
\text { and } \tilde{t}_{t r}=\langle 17.5,18.0,18.5\rangle \mathrm{min} .
\end{gathered}
$$

Because of the non-linearity of fuzzy arithmetic, the value of the excavator and truck productivity is an approximation of a triangular fuzzy number.

Finally, if the volume of the earthworks is $\langle 97000,100000,103000\rangle \mathrm{m}^{3}$, the swell factor is $\widetilde{\varepsilon}=\langle 1.55,1.60,1.65\rangle$, the operational efficiency is $\langle 0.72,0.75,0.78\rangle$, the contractual completion time is $6 * 20 * 8=960 \mathrm{hrs}(6$ months $)$, then the required productivity of the site is:

$$
\tilde{Q}_{w s}=\frac{\langle 97000,100000,103000\rangle \cdot\langle 1.55,1.60,1,65\rangle}{960 \cdot\langle 0.72,0.75,0.78\rangle}=\langle 200,222,245\rangle \mathrm{m}^{3} / \mathrm{h}
$$

Table 1. Fuzzy Productivity of a Caterpillar Excavator

\begin{tabular}{cc}
\hline Parameter & V alues \\
\hline bucket capacity $\left(\mathrm{m}^{3}\right)$ & 3 \\
fill factor & $\langle 0.81,0.83,0.84\rangle$ \\
cycle time $(\mathrm{min})$ & $\langle 0.51,0.52,0.54\rangle$ \\
operational efficiency & $\langle 0.78,0.80,0.81\rangle$ \\
excavator production rate $\left(\mathrm{m}^{3} / \mathrm{h}\right) \tilde{Q}_{\text {ex }}$ & $\langle 210,230,240\rangle$ \\
\hline
\end{tabular}


Table 2. Fuzzy Productivity of a Caterpillar Truck

\begin{tabular}{cc}
\hline Parameter & Values \\
\hline volume of transferred material $\left(\mathrm{m}^{3}\right)$ & $\langle 17.8,18.0,18.2\rangle$ \\
cycle time of truck $(\mathrm{min})$ & $\langle 17.5,18.0,18.5\rangle$ \\
operational efficiency & $\langle 0.69,0.75,0.81\rangle$ \\
fuzzy truck production rate $\left(\mathrm{m}^{3} / \mathrm{h}\right) \tilde{Q}_{t r}$ & $\langle 40,45,50\rangle$ \\
\hline
\end{tabular}

\subsection{Fleet Productivity}

Consider a fleet of excavators and trucks that have to complete an earthworks project. In applying uncertainty analysis, as shown above, it is possible to define the individual productivity rates with fuzzy numbers, i.e. $\tilde{Q}_{e x}=\langle 210,230,240\rangle \mathrm{m}^{3} / \mathrm{h}$ and $\tilde{Q}_{t r}=\langle 40,45,50\rangle \mathrm{m}^{3} / \mathrm{h}$. Furthermore, the fuzzy productivity of a homogeneous group of 5 similar trucks is $\tilde{Q}_{T R}=5 \cdot \tilde{Q}_{t r}=\langle 200,225,250\rangle \mathrm{m}^{3} / \mathrm{h}$. Understandably, as the number of trucks increases, so does the spread of the cumulative uncertainty of the group.

Table 3 shows the fleet productivity for some different compositions. In the issue of determining the critical machine, in case A that we have 1 excavator and 4 trucks and since $\widetilde{Q}_{T R}$ is definitely smaller than $\widetilde{Q}_{E X}$ it is evident that $\tilde{Q}_{\text {fleet }}=\widetilde{Q}_{T R}$ meaning that the trucks are always critical (Figure 1). Similarly, in the case of $\mathrm{C}$, if there are 6 trucks and 1 excavator, then the excavator is always critical. However, in the case of $\mathrm{B}$, the combination of 1 excavator and 5 trucks leads to an instance of composite criticality. Overall, in Figure 1, in cases $\mathrm{A}$ and $\mathrm{C}$, one type of machinery is definitely critical but there is also a waste of extra production capability since the total production is constrained by the lowest resource. In the case of $\mathrm{B}$, there is a better matching and balancing of the two types of machines even though it is not possible to determine which one is critical because of a situation of composite criticality.

Table 3. The productivity of a Fleet of Trucks and Excavators

\begin{tabular}{ccccccc}
\hline & $\tilde{Q}_{\text {fleet }}\left(\mathbf{m}^{3} / \mathbf{h}\right)$ & $\tilde{Q}_{T R}\left(\mathbf{m}^{3} / \mathbf{h}\right)$ & $\tilde{Q}_{E X}\left(\mathbf{m}^{3} / \mathbf{h}\right)$ & $n_{t r}$ & $n_{\text {ex }}$ & Criticality \\
\hline A & $\langle 160,180,220\rangle$ & $\langle 160,180,200\rangle$ & $\langle 210,230,240\rangle$ & 4 & 1 & trucks \\
B & composite & $\langle 200,225,250\rangle$ & $\langle 210,230,240\rangle$ & 5 & 1 & composite \\
C & $\langle 210,230,240\rangle$ & $\langle 240,270,300\rangle$ & $\langle 210,230,240\rangle$ & 6 & 1 & excavator \\
\hline
\end{tabular}



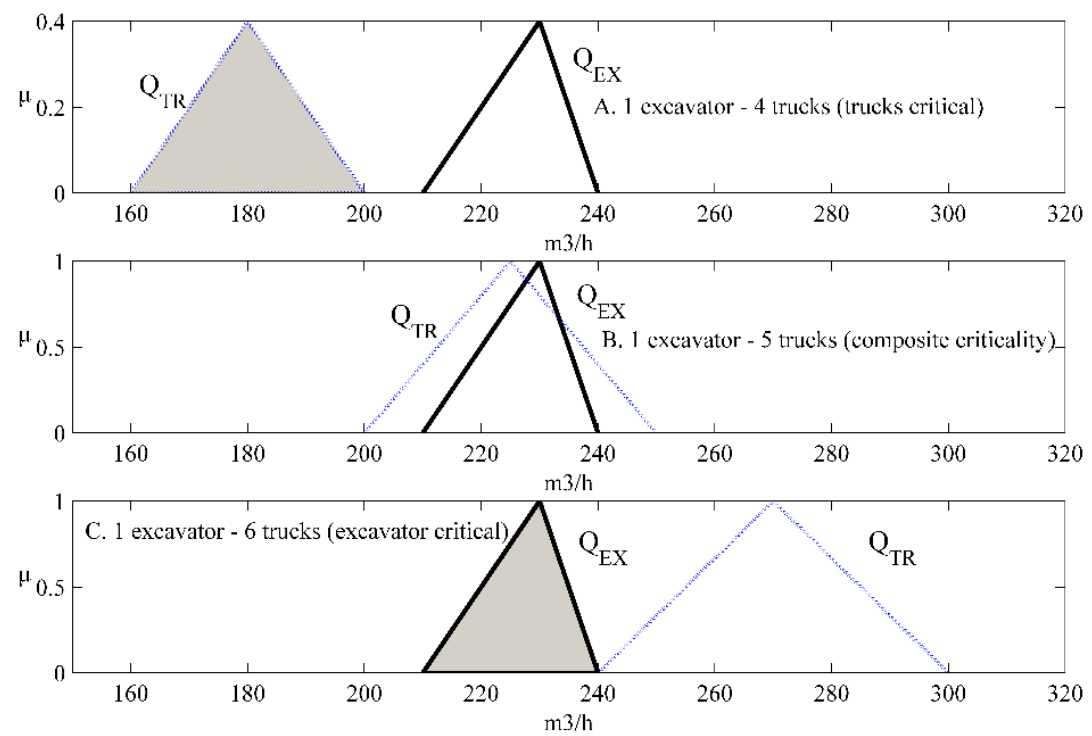

Fig. 1. Criticality in Different Fleet Compositions

Figure 2 helps us understand and interpret the situation of composite criticality. The single excavator has productivity of $\widetilde{Q}_{E X}=\langle 210,230,240\rangle \mathrm{m}^{3} / \mathrm{h}$ and the 5 trucks have $\tilde{Q}_{T R}=\langle 200,225,250\rangle \mathrm{m}^{3} / \mathrm{h}$. The resulting productivity of the fleet is depicted by the shaded area which is not a triangular fuzzy number. In the region of low risk (possibility level of 1 to that of 0.66) the trucks are critical since they have lower productivity. In the region of higher risk (possibility level of 0.66 to that of 0 ) the excavator is more likely to be critical because it has lower productivity. Physically, this means that for this particular combination in some cases the trucks are arriving at the loader faster than they are being served or the loaders are serving faster than the trucks arriving. Hence, contrary to deterministic analysis, no one type of machinery is definitely critical.

The agreement index for this fleet composition is:

$$
A I_{\text {fleet }}=A I\left(\tilde{Q}_{E X}, \widetilde{Q}_{T R}\right)=\left(\text { area } \tilde{Q}_{E X} \cap \widetilde{Q}_{T R}\right) /\left(\text { area } \tilde{Q}_{T R}\right)=21.65 / 25=0.86
$$

which can be interpreted as a relatively high degree of matching and balancing of the two types of machinery.

Figure 3 shows a graph of the fuzzy Match Factor which is calculated as:

$$
M \tilde{F}=\frac{\widetilde{Q}_{E X}}{\widetilde{Q}_{T R}}=\frac{\langle 210,230,240\rangle}{\langle 200,225,250\rangle}=\langle 0.84,1.02,1.20\rangle
$$

The value of the match factor shows that there is a high level of matching that is very close to the ideal value of 1 . Additionally, the match factor has a considerable spread of about $+/-18 \%$. Overall, in the presence of uncertainty, there are situations of composite criticality under which the productivity of the excavators is lower than that of the trucks and vice versa. The agreement index and the fuzzy match factor help in quantifying the degree of machinery matching. 


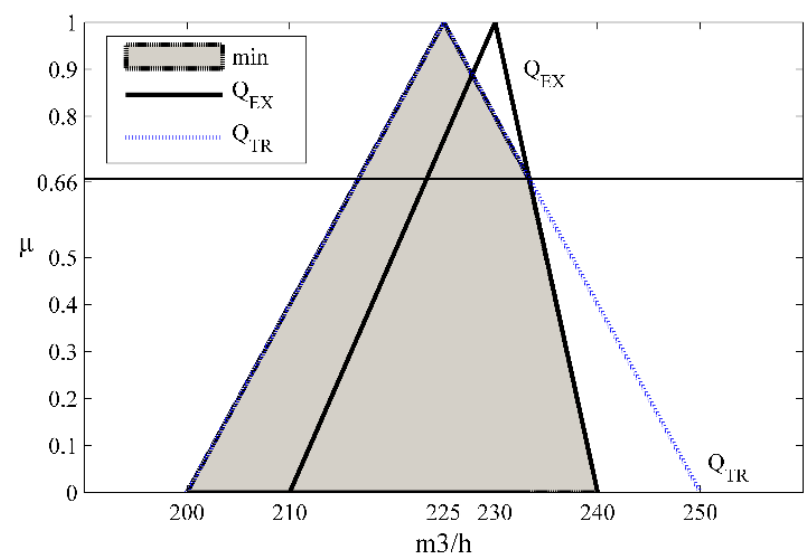

Fig. 2. Composite Criticality between Trucks and an Excavator

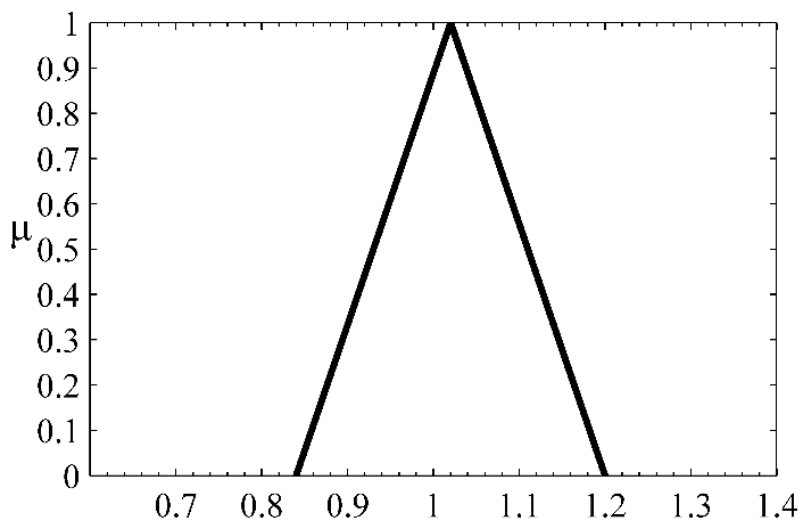

Fig. 3. Fuzzy M atch Factor

\section{Discussion and Conclusions}

At the level of individual machines, this paper shows how the fuzzy productivity of excavators and trucks can be estimated based on fuzzifying parameters such as the bucket fill factor, the cycle time and the operational efficiency. Thereafter, a methodology is presented in order to find the optimal composition of a fleet of machines that have uncertain production rates. In the process, the paper has challenged the well established deterministic assumption, that in a fleet of construction machines one type is definitely critical. On the contrary, if productivities are characterized by fuzzy numbers, in some cases it is difficult to determine which machine is critical because of composite criticality.

In the presence of uncertainty, a fuzzy match factor with a value near 1 and a small spread is an indication of a maximized production level. However, such a match factor, besides high efficiency, may also imply a situation of composite criticality because of the interference and overlapping of fuzziness. Similarly, a fleet Agreement Index close to 1 indicates optimal matching. In this particular example, composite criticality means that in some cases the trucks are arriving at the loader faster than they are being served and in others, the loaders are serving faster than the trucks arriving. Overall, when dealing with two different types 
of machinery in a fleet, it is usually required that their productivities are similar in order to reduce "waste" and idle times. The situation of composite criticality may actually be more efficient than having definite criticality.

While this paper has used an approach of applying fuzzy set theory to well-established methodologies in Performance Handbooks and traditional productivity theory equations, there may be potential in applying concepts presented in this paper to a fuzzy expert system regarding fleet management. Fuzzy expert systems, which can be useful in estimating productivity, are based on many fuzzy if-then rules that relate the input factors to the output factors. As illustrated by Fayek and Oduba [16] and Zhang et al. [17] to this extent a) system inputs would have to be defined b) linguistic descriptions of the inputs and numerical scales would have to be set c) the outputs would have to be defined and d) a detailed set of rules relating the inputs to the outputs would have to be presented.

Finally, additional future research can be directed into studying fuzzy costs along with fuzzy productivities. Thus, extensive search and optimization algorithms can be applied in determining the optimal composition and operation of construction fleets in the presence of uncertainty. Also, field testing of this theoretical model in an actual project may assist in verifying the concept. Overall, the proposed methodology can lead to a better understanding of productivity estimation, which in turn means better project scheduling and financial planning.

\section{References}

1. J.P. Pantouvakis, Data A nalysis and Design of Construction Productivity Efficiency Multipliers, In: Şahin S. (ed.) 8th International Conference on Engineering, Project, and Product Management (EPPM 2017. Lecture Notes in Mechanical Engineering. Cham: Springer, 119-128 (2018)

2. H. Park, Conceptual framework of construction productivity estimation, KSCE Journal of Civil Engineering 10(5):311-317 (2006)

3. Caterpillar, Caterpillar Performance Handbook (Edition 46), Illinois: Caterpillar Inc (2016)

4. K omatsu, Specifications and application handbook (Edition 31), Japan: K omatsu (2013)

5. R.L. Peurifoy, C.J. Schexnayder, A. Shapira, R. Schmitt, Construction Planning, Equipment and Methods (8th Edition), USA: M cGraw Hill (2010)

6. S.W. Nunally, Construction methods and management, Upper Saddle River: Prentice Hall (2007)

7. J. Douglas, Prediction of shovel-truck production: a reconciliation of computer and conventional estimates, Technical Report no. 37, Department of Civil Engineering, Stanford University (1964)

8. W. M organ, L. Peterson, Determining shovel-truck productivity, Mining Engineering 76 -80 (1968)

9. C.N. Burt, L. Caccetta, Match factor for heterogeneous truck and loader fleets, International Journal of Mining, Reclamation and Environment 21(4):262-270 (2007)

10. K aufman, M.M. Gupta, Introduction to fuzzy arithmetic, A msterdam: N orth Holland (1985)

11. L .A . Zadeh, The Concept of a Linguistic V ariable and its A pplication to A pproximate Reasoning - II, Information Sciences 8:301-357 (1975)

12. T.J. Ross, Fuzzy Logic with Engineering Applications (2nd Edition), Chichester-West Sussex, England: W iley (2004)

13. C.S. McCahon, E.S. Lee, Project Network Analysis with Fuzzy Activity Times, Computers and Mathematics with Applications 15(10):829-838 (1988)

14. Maravas, J.P. Pantouvakis, A Process for the Estimation of the Duration of A ctivities in Fuzzy Project Scheduling, In: A yyub B.M. (ed.), Vulnerability, Uncertainty and Risk 
/ Analysis, Modeling and Management - Proceedings of the first International Conference on Vulnerability and Risk Analysis and Management (ICVRAM 2011) and the fifth International Symposium on Uncertainty Modeling and Analysis (ISUMA 2011) 11-13, M aryland. USA : A SCE, 62-69 (2011)

15. M aravas, Scheduling and Financial Planning of Projects and Portfolios with Fuzzy Constraints, Thesis (PhD), National Technical University of A thens, A thens (2016)

16. A.R. Fayek, A. Oduba, Predicting industrial construction labor productivity using fuzzy expert systems, Journal of Construction Engineering and Management 131(8):938-941 (2005)

17. H. Zhang, C.M. Tam, J.J. Shi, A pplication of fuzzy logic to simulation for construction operations, Journal of Computing in Civil Engineering 17(1):38-45 (2003) 